\title{
Asteroseismic analysis of solar-mass subgiants KIC 6442183 and KIC 11137075 observed by Kepler
}

\author{
Zhijia Tian ${ }^{1}$, Shaolan $\mathrm{Bi}^{1}$, Timothy R. Bedding ${ }^{2,3}$, and Wuming Yang ${ }^{1}$ \\ ${ }^{1}$ Department of Astronomy, Beijing Normal University, 100875 Beijing, PR China \\ e-mail: tianzhijia@mail.bnu.edu.cn; bisl@bnu.edu.cn \\ 2 Sydney Institute for Astronomy (SIfA), School of Physics, University of Sydney, NSW 2006, Australia \\ e-mail: t . bedding@physics.usyd.edu.au \\ 3 Stellar Astrophysics Centre, Department of Physics and Astronomy, Aarhus University, Ny Munkegade 120, \\ 8000 Aarhus C, Denmark
}

Received 26 February 2015 / Accepted 1 June 2015

\section{ABSTRACT}

\begin{abstract}
Context. Asteroseismology provides a powerful way to constrain stellar parameters. Solar-like oscillations have been observed on subgiant stars with the Kepler mission. The continuous and high-precision time series enables us to carry out a detailed asteroseismic study for these stars.

Aims. We carry out data processing of two subgiants of spectral type G: KIC 6442183 and KIC 11137075 observed with the Kepler mission, and perform seismic analysis for the two evolved stars.

Methods. We estimated the global asteroseismic parameters $\Delta v=64.9 \pm 0.2 \mu \mathrm{Hz}$ and $v_{\max }=1225 \pm 17 \mu \mathrm{Hz}$ for KIC 6442183, $\Delta v=65.5 \pm 0.2 \mu \mathrm{Hz}$ and $v_{\max }=1171 \pm 8 \mu \mathrm{Hz}$ for KIC 11137075 , respectively. In addition, we extracted the individual mode frequencies of the two stars. We compared stellar models and observations, including mode frequencies and mode inertias. The mode inertias of mixed modes, which are sensitive to the stellar interior, were used to constrain stellar models. We defined a quantity $d v_{m-p}$ that measures the difference between the mixed modes and the expected pure pressure modes, which is related to the inertia ratio of mixed modes to radial modes.

Results. Asteroseismic together with spectroscopic constraints provide the estimates of the stellar parameters: $M=1.04_{-0.04}^{+0.01} M_{\odot}$, $R=1.66_{-0.02}^{+0.01} R_{\odot}$ and $t=8.65_{-0.06}^{+1.12} \mathrm{Gyr}$ for KIC 6442183, and $M=1.00_{-0.01}^{+0.01} M_{\odot}, R=1.63_{-0.01}^{+0.01} R_{\odot}$ and $t=10.36_{-0.20}^{+0.01} \mathrm{Gyr}$ for KIC 11137075. Either mode inertias or $d v_{m-p}$ can be used to constrain stellar models.
\end{abstract}

Key words. stars: individual: KIC 6442183 - stars: individual: KIC 11137075 - stars: oscillations - stars: fundamental parameters

\section{Introduction}

Asteroseismology provides a useful tool to probe stellar interiors, test internal physical processes and obtain stellar parameters accurately (e.g. Eggenberger et al. 2004; Bi et al. 2008; Kallinger et al. 2010; Montalbán et al. 2013). Many solar-like stars have been observed continuously and precisely with space missions such as CoRoT and Kepler (Appourchaux et al. 2008; Borucki et al. 2007), ushering in a golden age for the asteroseismic analysis of oscillating stars.

The oscillations of evolved stars include mixed modes, which behave as pressure modes ( $p$-modes) in the envelope and gravity modes ( $g$-modes) in the core (Osaki 1975; Aizenman et al. 1977). Mixed modes have been used to distinguish between red clump stars and red giant branch (RGB) stars (Bedding et al. 2011; Mosser et al. 2011a), and to monitor the stellar evolution status from the main sequence (MS) to the asymptotic giant branch (Mosser et al. 2014). The signature of avoided crossings raises the exciting possibility that detailed modeling of the star will provide a very precise determination of its age (Gilliland et al. 2010a; Chaplin et al. 2010; Metcalfe et al. 2010; Benomar et al. 2012; Bedding 2014).

As discussed by Dziembowski et al. (2001), Christensen-Dalsgaard (2004), Dupret et al. (2009), Benomar et al. (2014) and Datta et al. (2015), the $g$-dominated non-radial modes have much higher inertia than the radial modes, whereas the $p$-dominated modes do not. Because the mixed modes possess the properties of partial $g$-modes, the inertia of mixed modes is usually higher than that of $p$-modes. The inertia of mixed modes could provide powerful constraints on stellar models.

Subgiants are the bridge between the MS and red giants on the Hertzsprung-Russel (H-R) diagram. The study of subgiants could provide valuable insight into stellar structure and evolution. When stars leave the MS, the $g$-mode and $p$-mode frequencies overlap, which causes mixed modes in post-MS stars. KIC 6442183 (also known as "Dougal") and KIC 11137075 (also known as "Zebedee") are at the beginning of the subgiant stage. The magnitudes of the stars are 8.52 and 10.86 (KIC magnitude), which are bright enough to allow seismic observations. They have been continuously observed by the Kepler mission with a short cadence of $58.84 \mathrm{~s}$ (Gilliland et al. 2010b) for quarters 6.1-17.2 and 7.1-11.3, respectively. Benomar et al. $(2013,2014)$ extracted the mode frequencies and measured the mode inertia ratio for KIC 6442183 . They estimated a mass $M=0.94 M_{\odot}$ and a radius $R=1.60 R_{\odot}$ for KIC 6442183 using scaling relations (Brown et al. 1991; Kjeldsen \& Bedding 1995). With oscillation observations from Kepler and spectroscopic observations from Bruntt et al. (2012) and Molenda-Żakowicz et al. (2013), we can now conduct asteroseismic analyses and can accurately constrain stellar parameters for the two stars.

In Sect. 2, we review the recent observations and extract the mode frequencies for the two stars. In Sect. 3, we construct 
Table 1. Basic parameters of KIC 6442183 and KIC 11137075 from observations.

\begin{tabular}{|c|c|c|}
\hline & KIC 6442183 & KIC 11137075 \\
\hline$T_{\text {eff }}(\mathrm{K})$ & $5740 \pm 70^{a} \quad 5738 \pm 62^{b}$ & $5590 \pm 70^{a} \quad 5610 \pm 71^{b}$ \\
\hline $\log g(\operatorname{dex})$ & $4.03 \pm 0.03^{a} \quad 4.14 \pm 0.10^{b}$ & $4.01 \pm 0.03^{a} \quad 4.10 \pm 0.12^{b}$ \\
\hline$[\mathrm{Fe} / \mathrm{H}]$ & $-0.11 \pm 0.06^{a}-0.12 \pm 0.05^{b}$ & $-0.06 \pm 0.06^{a}-0.06 \pm 0.06^{b}$ \\
\hline KIC magnitude & $8.52^{e}$ & $10.86^{e}$ \\
\hline$\Delta v(\mu \mathrm{Hz})$ & $65.07 \pm 0.09^{c} \quad 64.9 \pm 0.2^{d}$ & $65.5 \pm 0.2^{d}$ \\
\hline$v_{\max }(\mu \mathrm{Hz})$ & $1160 \pm 4^{c} \quad 1225 \pm 17^{d}$ & $1171 \pm 8^{d}$ \\
\hline$M\left(M_{\odot}\right)$ & $0.94^{c} \quad 1.04_{-0.04}^{+0.01 d}$ & $1.00_{-0.01}^{+0.01 d}$ \\
\hline$R\left(R_{\odot}\right)$ & $1.60^{c} \quad 1.66_{-0.02}^{+0.01 d}$ & $1.63_{-0.01}^{+0.01 d}$ \\
\hline
\end{tabular}

Notes. ${ }^{(a)}$ Bruntt et al. (2012); ${ }^{(b)}$ Molenda-Żakowicz et al. (2013); ${ }^{(c)}$ Benomar et al. (2013), who stated that typical uncertainties on mass and radius were a few percent; ${ }^{(d)}$ this work; ${ }^{(e)}$ Kepler Asteroseismic Science Operations Center: http://kasoc.phys .au.dk/

stellar models and correct the near-surface term for the theoretical frequencies of the models. Seismic analyses and stellar parameter determinations are shown in Sect. 4. Finally, the discussions and conclusions are presented in Sect. 5.

\section{Observations and data processing}

Spectroscopic observations from Bruntt et al. (2012) and Molenda-Żakowicz et al. (2013) provided the values of metallicity $([\mathrm{Fe} / \mathrm{H}])$, effective temperature $\left(T_{\text {eff }}\right)$ and gravity $(\log g)$ for these two stars. Results from the two groups show good consistency, and yield preliminary constraints on the stars. We adopted the parameters obtained by Bruntt et al. (2012) because they took the asteroseismic $\log g$ into account during the spectroscopic analysis.

We obtained the power spectra by applying the LombScargle periodogram (Lomb 1976; Scargle 1982) to the shortcadence time series corrected by the KASC Working Group 1 (WG\#1; "solar-like oscillating stars") following García et al. (2011), which is available to the Kepler Asteroseismic Science Consortium (KASC; Kjeldsen et al. 2010) through the KASOC database ${ }^{1}$.

The autocorrelation of the power spectrum can provide the estimate of periodic information, such as the mean large frequency separation (Barban et al. 2009). We estimated the large frequency separations $\Delta v$ and the frequency of maximum power $v_{\max }$ with the autocorrelation function (e.g. Roxburgh \& Vorontsov 2006; Roxburgh 2009; Mosser \& Appourchaux 2009) and the collapsed autocorrelation function (e.g. Huber et al. 2009; Tian et al. 2014). These global oscillation parameters were evaluated to be $\Delta v=64.9 \pm 0.2 \mu \mathrm{Hz}$ and $v_{\max }=$ $1225 \pm 17 \mu \mathrm{Hz}$ for KIC 6442183, and $\Delta v=65.5 \pm 0.2 \mu \mathrm{Hz}$ and $v_{\max }=1171 \pm 8 \mu \mathrm{Hz}$ for KIC 11137075 . The global oscillation parameters $\left(\Delta v\right.$ and $\left.v_{\max }\right)$ and atmospheric constraints for the two stars are listed in Table 1.

As discussed by Mathur et al. (2011), several methods have been developed to extract individual frequencies, and the key point is to fit a sum of Lorentzian profiles describing all oscillation modes. We followed the method described by Basu et al. (2000) and Régulo \& Roca Cortés (2007), where each oscillation mode in the power spectrum was fitted to a Lorentzian profile using a robust non-linear least squares method (Markwardt 2009, 2012). As discussed by Appourchaux et al. (1998a,b), the noise statistics of each bin of the power spectrum has a $\chi^{2}$ distribution

\footnotetext{
1 Kepler Asteroseismic Science Operations Center: http://kasoc. phys.au.dk/.
}

with two degrees of freedom. However, the non-linear least squares method assumes that this statistics is Gaussian. The mode extraction method adopted in this work might introduce a certain bias of amplitudes and linewidths, and fortunately does not affect the frequencies. To estimate the errors, we measured the individual frequencies quarter by quarter, and estimated the frequency and error of each mode by combining each subset. Asymptotic formulae show that the oscillation mode linewidthds are related to the $T_{\text {eff }}$ of stars with a power law (Chaplin et al. 2009; Appourchaux et al. 2012). The smallest linewidth of the detected modes for KIC 6442183 is of approximately $0.25 \mu \mathrm{Hz}$ (Benomar et al. 2013). Because the difference of $T_{\text {eff }}$ between the two subgiants is about $150 \mathrm{~K}$, they will obtain the linewidths of the same magnitude. Therefore, the frequency resolution of a Kepler quarter $(0.1286 \mu \mathrm{Hz})$ is sufficient to resolve the oscillation modes for the two stars.

This procedure provided independently obtained frequency sets with symmetric errors. We obtained 37 oscillation modes of degree $l=0-3$ for KIC 6442183 and 26 oscillation modes of degree $l=0-2$ for KIC 11137075 . The individual frequencies for the two stars are listed in Tables 2 and 3. The oscillation frequencies for KIC 6442183 in this work and those given by Benomar et al. (2013) are consistent at the $1 \sigma$ level. The mode frequencies of the power spectra are overplotted in the échelle diagram in Figs. 3 and 4. The radial modes follow a vertical ridge in the figures, and the non-radial modes show avoided crossings, especially for the $l=1$ modes. In addition to the $l=0-2$ modes, there are five $l=3$ modes identified for KIC 6442183, which will provide extra constraints on theoretical models.

\section{Stellar models}

To estimate the parameters of the two stars, a grid of stellar evolutionary models was constructed with the Yale stellar evolution code (YREC7; Demarque et al. 2008). We used the OPAL opacity table GN93 (Iglesias \& Rogers 1996), the low-temperature table AGS05 (Ferguson et al. 2005), the OPAL equation-of-state tables EOS2005 (Rogers \& Nayfonov 2002), and the Bahcall nuclear rates (Bahcall et al. 1995) for microphysics. We chose the Eddington grey atmosphere $T-\tau$ relation. We adopted the standard mixing-length theory (Böhm-Vitense 1958) and overshooting to treat convection. The coefficient of helium and heavy elements diffusion was taken from Thoul et al. (1994). We did not take rotation or magnetic field into consideration in our calculations. 
Table 2. Observed frequencies for KIC 6442183.

\begin{tabular}{|c|c|c|}
\hline$l$ & Frequencies $(\mu \mathrm{Hz})$ & Error $(\mu \mathrm{Hz})$ \\
\hline 0 & 809.63 & 0.11 \\
\hline 0 & 874.08 & 0.25 \\
\hline 0 & 937.21 & 0.10 \\
\hline 0 & 1001.04 & 0.12 \\
\hline 0 & 1065.65 & 0.14 \\
\hline 0 & 1130.50 & 0.09 \\
\hline 0 & 1195.40 & 0.03 \\
\hline 0 & 1260.32 & 0.12 \\
\hline 0 & 1325.73 & 0.19 \\
\hline 0 & 1391.69 & 0.55 \\
\hline 1 & 783.35 & 0.31 \\
\hline 1 & 840.62 & 0.11 \\
\hline 1 & 901.27 & 0.14 \\
\hline 1 & 960.26 & 0.09 \\
\hline 1 & 1002.91 & 0.21 \\
\hline 1 & 1037.60 & 0.09 \\
\hline 1 & 1096.83 & 0.25 \\
\hline 1 & 1159.96 & 0.06 \\
\hline 1 & 1224.09 & 0.13 \\
\hline 1 & 1288.75 & 0.22 \\
\hline 1 & 1354.10 & 0.26 \\
\hline 1 & 1419.52 & 0.37 \\
\hline 2 & 801.63 & 0.35 \\
\hline 2 & 868.11 & 0.17 \\
\hline 2 & 931.16 & 0.30 \\
\hline 2 & 996.92 & 0.15 \\
\hline 2 & 1059.95 & 0.17 \\
\hline 2 & 1124.73 & 0.23 \\
\hline 2 & 1189.88 & 0.15 \\
\hline 2 & 1254.61 & 0.14 \\
\hline 2 & 1321.26 & 0.31 \\
\hline 2 & 1386.81 & 0.41 \\
\hline 3 & 1150.13 & 0.25 \\
\hline 3 & 1215.93 & 0.31 \\
\hline 3 & 1280.49 & 0.31 \\
\hline 3 & 1347.26 & 0.37 \\
\hline 3 & 1412.36 & 0.59 \\
\hline
\end{tabular}

The ratio of heavy elements to hydrogen as a mass fraction was estimated through the formula

$[\mathrm{Fe} / \mathrm{H}]=\log \left(\frac{Z}{X}\right)-\log \left(\frac{Z}{X}\right)_{\odot}$,

where we adopted the value of $\left(\frac{Z}{X}\right)_{\odot}=0.0245$ (Iglesias \& Rogers 1996) and the values of $[\mathrm{Fe} / \mathrm{H}]$ from Bruntt et al. (2012) for the two stars. The abundance ratio of the heavy elements to hydrogen $\left(\frac{Z}{X}\right)_{\mathrm{s}}$ is in the range of $0.0165-0.0218$ and $0.0186-$ 0.0245 for KIC 6442183 and KIC 11137075 , respectively. In the model calculation, we chose the initial helium abundance as $Y_{i}=0.245+1.54 Z_{i}$ (e.g., Dotter et al. 2008; Thompson et al. 2014 ) in terms of the initial metal abundance. For a given mass, stellar evolutionary models depend on three free parameters: initial chemical compositions, the mixing-length parameter $\alpha$, and the overshooting parameter $\alpha_{\mathrm{ov}}$. The input parameters for the model calculations are listed in Table 4.

We calculated three groups of tracks for these two stars: (i) models without diffusion and without overshooting; (ii) models with diffusion and without overshooting; and (iii) models with diffusion and overshooting $\left(\alpha_{\mathrm{ov}}=0.2\right)$. Because the observed luminosities of the two stars were not available, we used the large frequency separation $\Delta v$ to constrain stellar models in
Table 3. Observed frequencies for KIC 11137075.

\begin{tabular}{ccc}
\hline \hline$l$ & Frequencies $(\mu \mathrm{Hz})$ & Error $(\mu \mathrm{Hz})$ \\
\hline 0 & 949.02 & 0.22 \\
0 & 1013.37 & 0.22 \\
0 & 1078.56 & 0.09 \\
0 & 1144.34 & 0.10 \\
0 & 1209.66 & 0.13 \\
0 & 1275.11 & 0.08 \\
0 & 1341.11 & 0.25 \\
0 & 1407.67 & 0.45 \\
\hline 1 & 916.94 & 0.33 \\
1 & 978.01 & 0.11 \\
1 & 1040.74 & 0.10 \\
1 & 1101.50 & 0.10 \\
1 & 1142.65 & 0.12 \\
1 & 1180.26 & 0.09 \\
1 & 1240.80 & 0.07 \\
1 & 1304.94 & 0.19 \\
1 & 1370.07 & 0.62 \\
1 & 1436.91 & 1.05 \\
\hline 2 & 943.76 & 0.47 \\
2 & 1007.47 & 0.21 \\
2 & 1072.59 & 0.21 \\
2 & 1132.52 & 0.17 \\
2 & 1141.38 & 0.23 \\
2 & 1204.19 & 0.07 \\
2 & 1269.64 & 0.20 \\
2 & 1335.61 & 0.73 \\
\hline
\end{tabular}

Table 4. Input parameters for the model calculations.

\begin{tabular}{lcc}
\hline \hline Variable & Range & $\delta$ \\
\hline Mass $\left(M_{\odot}\right)$ & $0.90-1.08$ & 0.01 \\
$Z_{i}$ & $0.012-0.018$ & 0.001 \\
$\alpha$ & $1.70-1.90$ & 0.2 \\
$\alpha_{\text {ov }}$ & $0.0-0.2$ & 0.2 \\
\hline
\end{tabular}

the H-R diagram. Considering the difference between $\Delta v$ of the models from the scaling relation and pulsation code, we assigned an error of approximately $2 \mu \mathrm{Hz}$ to the large separation in the $\mathrm{H}-\mathrm{R}$ diagram. There are 256 and 265 tracks falling into their error boxes for KIC 6442183 and KIC 11137075, respectively, as shown in Fig. 5.

\section{Asteroseismic diagnostics}

\subsection{Model calibration}

For stellar models along the tracks falling inside the error box, we computed smaller and more finely sampled grids around these models and calculated the theoretical mode frequencies with Guenther's pulsation code (Guenther 1994). It is well known that there is a systematic offset between observed and computed frequencies that arises from improper modeling of the near-surface layers for both the Sun and solar-type stars (Kjeldsen et al. 2008, and references therein). To correct the near-surface term, we followed the method described by Brandão et al. (2011), who corrected the near-surface term for radial modes with the method proposed by Kjeldsen et al. (2008) by fitting a power law,

$v_{\mathrm{obs}}(n, 0)-v_{\text {best }}(n, 0)=a\left(\frac{v_{\mathrm{obs}}(n, 0)}{v_{0}}\right)^{b}$. 


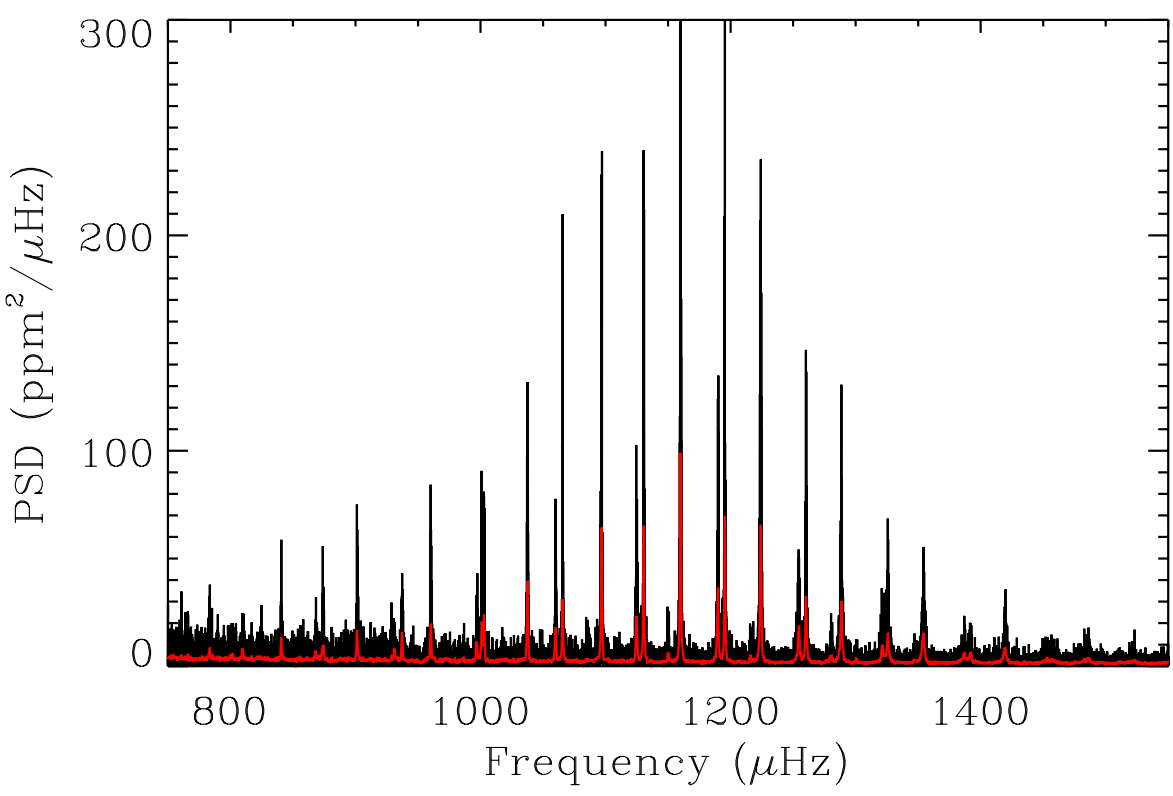

Fig. 1. Power spectrum for KIC 6442183. The black and red lines denote the power spectrum before and after smoothing to $2 \mu \mathrm{Hz}$.

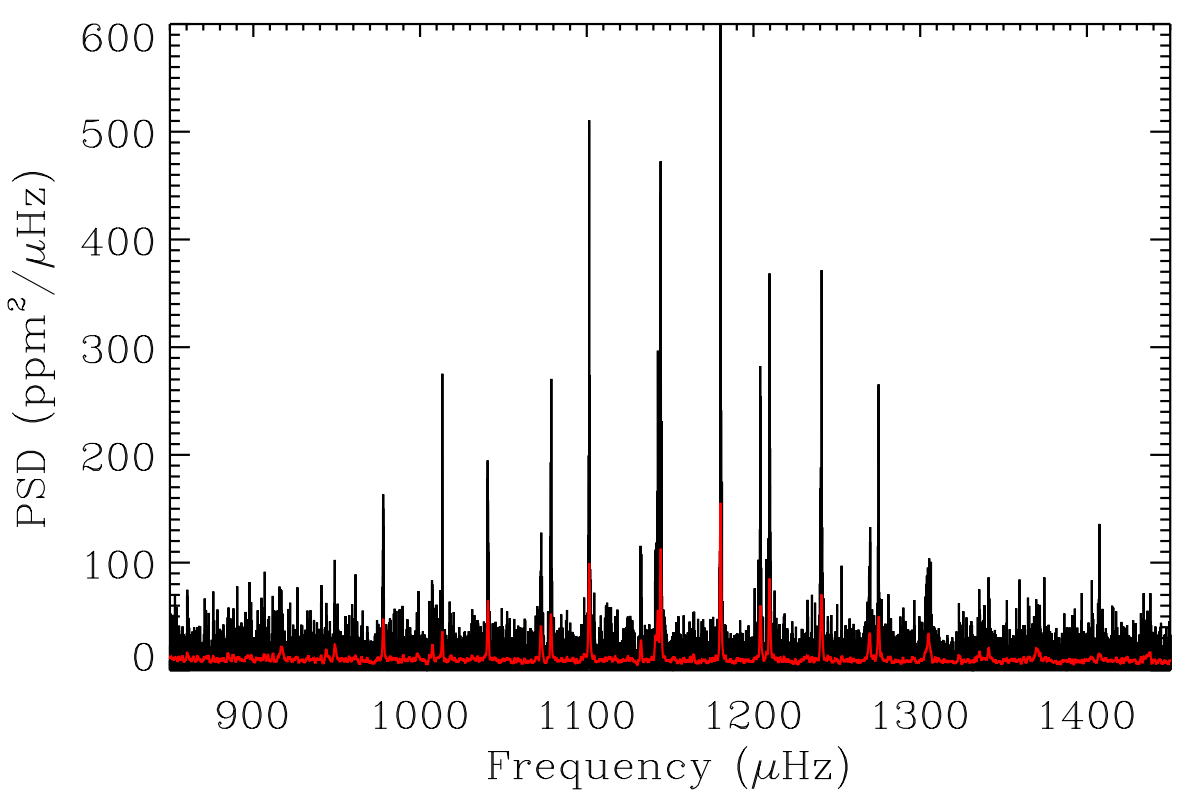

Fig. 2. Same as Fig. 1, but for KIC 11137075.

For the mixed modes, the corrected frequencies $v_{\text {corr }}(n, l)$ were calculated through:

$v_{\text {corr }}(n, l)=v_{\text {best }}(n, l)+a\left(\frac{1}{Q_{n l}}\right)\left(\frac{v_{\mathrm{obs}}(n, l)}{v_{0}}\right)^{b}$,

where $n$ and $l$ are the order and degree of the modes, respectively, $b$ is the exponent calibrated from the solar models, and $a$ is a constant for the stellar model. The quantity $Q_{n l}$ presents the ratio between the inertia of the non-radial mode $I_{l}$ and the inertia of a radial mode $I_{0}$ of the same frequency (Aerts et al. 2010). The inertia of mode $I_{l}$ is defined as:

$I_{l}=4 \pi \int_{0}^{R}\left[\left|\xi_{r}(r)\right|^{2}+l(l+1)\left|\xi_{h}(r)\right|^{2}\right] \rho_{0} r^{2} \mathrm{~d} r$

where $\xi_{r}(r)$ and $\xi_{h}(r)$ are the radial and horizontal displacements of the modes at the radius $r$, respectively. The inertia ratio between non-radial and radial modes can be expressed as

$Q_{n l}=I_{l} / I_{0} \simeq 1+\frac{l(l+1) \int_{0}^{R}\left|\xi_{h}(r)\right|^{2} \rho_{0} r^{2} \mathrm{~d} r}{\int_{0}^{R}\left|\xi_{r}(r)\right|^{2} \rho_{0} r^{2} \mathrm{~d} r}$.

As discussed by Aerts et al. (2010), the typical values for $\xi_{h}(R) / \xi_{r}(R)$ are $0.01-0.1$ for low-order $p$-modes, and 10-100 for high-order $g$-modes. This indicates that $Q_{n l} \simeq 1$ for pure acoustic modes and $Q_{n l}>1$ for $p-g$ mixed modes.

To restrict the stellar parameters, we performed a $\chi_{C}^{2}$ minimization by a comparison of models with observations,

$\chi_{C}^{2}=\frac{1}{3} \sum_{i=1}^{3}\left(\frac{C_{i}^{\text {theo }}-C_{i}^{\text {obs }}}{\sigma_{C_{i}}^{\text {obs }}}\right)^{2}$

where $C=\left(T_{\text {eff }},[\mathrm{Fe} / \mathrm{H}], \Delta v\right)$ and the $\sigma_{C_{i}}^{\text {obs }}$ denote the observational errors. We chose models with $\chi_{C}^{2}<1$ as candidates for 
Table 5. Best-candidate models for the two stars.

\begin{tabular}{lccccccccccccrr}
\hline \hline Model & $\begin{array}{l}\text { Mass } \\
\left(M_{\odot}\right)\end{array}$ & $\alpha$ & Diff & $Z_{i}$ & $\begin{array}{c}t \\
(\mathrm{Gyr})\end{array}$ & $(\mathrm{Z} / \mathrm{X})_{\mathrm{s}}$ & $\begin{array}{c}T_{\text {eff }} \\
(\mathrm{K})\end{array}$ & $\begin{array}{c}L \\
\left(L_{\odot}\right)\end{array}$ & $\begin{array}{c}R \\
\left(R_{\odot}\right)\end{array}$ & $\begin{array}{l}\log g \\
(\mathrm{dex})\end{array}$ & $\begin{array}{c}\Delta v \\
(\mu \mathrm{Hz})\end{array}$ & $\chi_{C}^{2}$ & $\chi_{v, l=0}^{2}$ & $\chi_{v, \text { all }}^{2}$ \\
\hline & & & & & & \multicolumn{1}{c}{ KIC 6442183} \\
\hline 1 & 0.98 & 1.70 & No & 0.012 & 10.06 & 0.017 & 5703 & 2.51 & 1.63 & 4.01 & 64.86 & 0.44 & 29.85 & 2514.27 \\
2 & 0.99 & 1.70 & No & 0.013 & 9.95 & 0.018 & 5685 & 2.50 & 1.63 & 4.01 & 64.85 & 0.27 & 28.32 & 599.80 \\
$\mathbf{3}$ & 1.00 & 1.70 & No & 0.014 & 9.80 & 0.019 & 5672 & 2.49 & 1.64 & 4.01 & 64.85 & 0.34 & 27.24 & 77.20 \\
$\mathbf{4}$ & 1.04 & 1.90 & No & 0.015 & 8.62 & 0.021 & 5807 & 2.82 & 1.66 & 4.01 & 64.85 & 0.49 & 26.46 & 70.98 \\
\hline 5 & 1.03 & 1.90 & Yes & 0.016 & 8.85 & 0.019 & 5709 & 2.62 & 1.66 & 4.01 & 64.84 & 0.09 & 22.40 & 1429.14 \\
6 & 1.04 & 1.90 & Yes & 0.015 & 8.30 & 0.017 & 5783 & 2.77 & 1.66 & 4.01 & 64.84 & 0.31 & 27.61 & 2208.02 \\
7 & 1.04 & 1.90 & Yes & 0.016 & 8.50 & 0.019 & 5743 & 2.69 & 1.66 & 4.01 & 64.84 & 0.03 & 23.46 & 532.24 \\
$\mathbf{8}$ & 1.04 & 1.90 & Yes & 0.017 & 8.68 & 0.020 & 5707 & 2.63 & 1.66 & 4.01 & 64.84 & 0.15 & 22.04 & 46.23 \\
9 & 1.04 & 1.90 & Yes & 0.018 & 8.85 & 0.021 & 5673 & 2.57 & 1.66 & 4.01 & 64.84 & 0.57 & 20.54 & 571.86 \\
10 & 1.05 & 1.90 & Yes & 0.018 & 8.50 & 0.021 & 5707 & 2.65 & 1.67 & 4.02 & 64.85 & 0.32 & 23.59 & 1480.05 \\
\hline & & & & & & KIC 11137075 & & & & & & \\
\hline 1 & 0.96 & 1.70 & No & 0.014 & 11.60 & 0.019 & 5549 & 2.19 & 1.60 & 4.01 & 65.50 & 0.26 & 81.36 & 1292.34 \\
2 & 1.00 & 1.90 & No & 0.015 & 10.14 & 0.021 & 5686 & 2.49 & 1.63 & 4.02 & 65.51 & 0.64 & 64.19 & 470.62 \\
$\mathbf{3}$ & 1.00 & 1.90 & No & 0.016 & 10.39 & 0.022 & 5645 & 2.42 & 1.63 & 4.01 & 65.52 & 0.26 & 43.86 & 39.74 \\
4 & 1.01 & 1.90 & No & 0.017 & 10.17 & 0.024 & 5645 & 2.43 & 1.63 & 4.02 & 65.53 & 0.44 & 19.19 & 839.92 \\
\hline $\mathbf{5}$ & 0.99 & 1.90 & Yes & 0.016 & 10.37 & 0.019 & 5587 & 2.30 & 1.62 & 4.01 & 65.53 & 0.25 & 19.16 & 111.69 \\
6 & 1.00 & 1.90 & Yes & 0.016 & 9.96 & 0.019 & 5622 & 2.38 & 1.63 & 4.01 & 65.48 & 0.32 & 135.53 & 1712.46 \\
7 & 1.00 & 1.90 & Yes & 0.017 & 10.16 & 0.020 & 5586 & 2.32 & 1.63 & 4.01 & 65.50 & 0.05 & 65.69 & 431.52 \\
$\mathbf{8}$ & 1.00 & 1.90 & Yes & 0.018 & 10.36 & 0.022 & 5553 & 2.26 & 1.63 & 4.01 & 65.51 & 0.10 & 44.50 & 32.11 \\
\hline
\end{tabular}

Notes. The input parameters were: initial mass, mixing-length parameter $(\alpha)$, inclusion of diffusion (Diff), and initial metal abundance $\left(Z_{i}\right)$. The model parameters were age $(t)$, surface abundance ratio $\left((Z / X)_{\mathrm{s}}\right)$, effective temperature $\left(T_{\text {eff }}\right)$, luminosity $(L)$, radius $(R)$, gravity $(\log g)$, mean large separation $(\Delta v), \chi_{C}^{2}, \chi_{v, l=0}^{2}$ and $\chi_{v, \text { all }}^{2}($ Cols. 6-15).

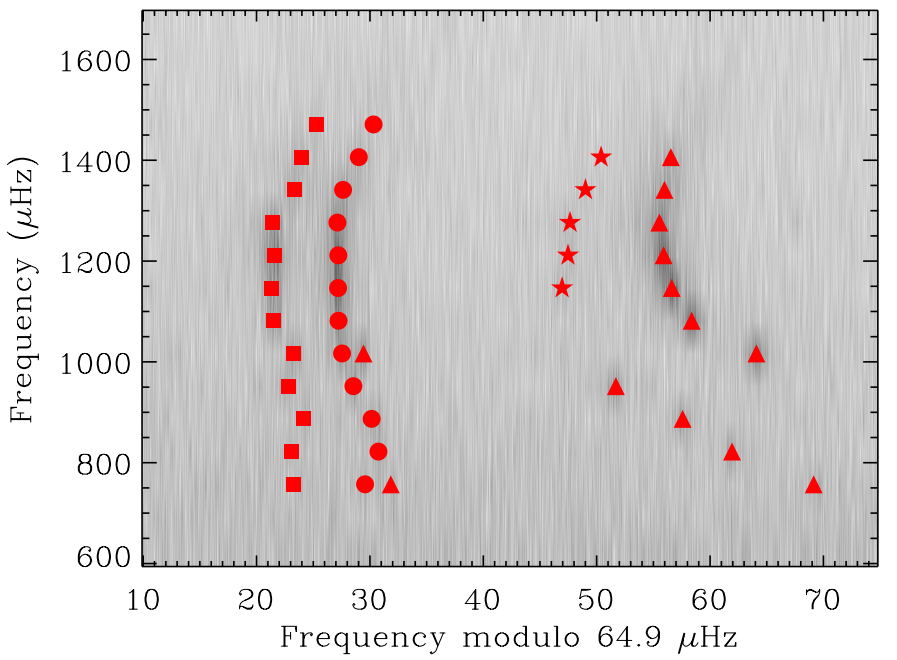

Fig. 3. Echelle diagram with the identified modes for KIC 6442183. The circles, triangles, squares and five-pointed stars show the frequencies of modes with $l=0,1,2$ and 3 .

the subsequent pulsation analysis. In addition to the constraints from the atmospheric parameters and $\Delta v$, we performed another $\chi_{v}^{2}$ minimization by a comparison of the near-surface-corrected model frequencies with the observed frequencies:

$\chi_{v}^{2}=\frac{1}{N} \sum_{i=1}^{N}\left(\frac{v_{i}^{\text {theo }}-v_{i}^{\text {obs }}}{\sigma_{v_{i}}^{\text {obs }}}\right)^{2}$,

where the superscripts "obs" and "theo" correspond to individual frequencies from observations and modeling, and $\sigma_{v_{i}}^{\text {obs }}$ denotes the observational errors. We list the candidate models with $\chi_{v, \text { all }}^{2}<3000$ for KIC 6442183 in Table 5. Since the $l=3$ modes

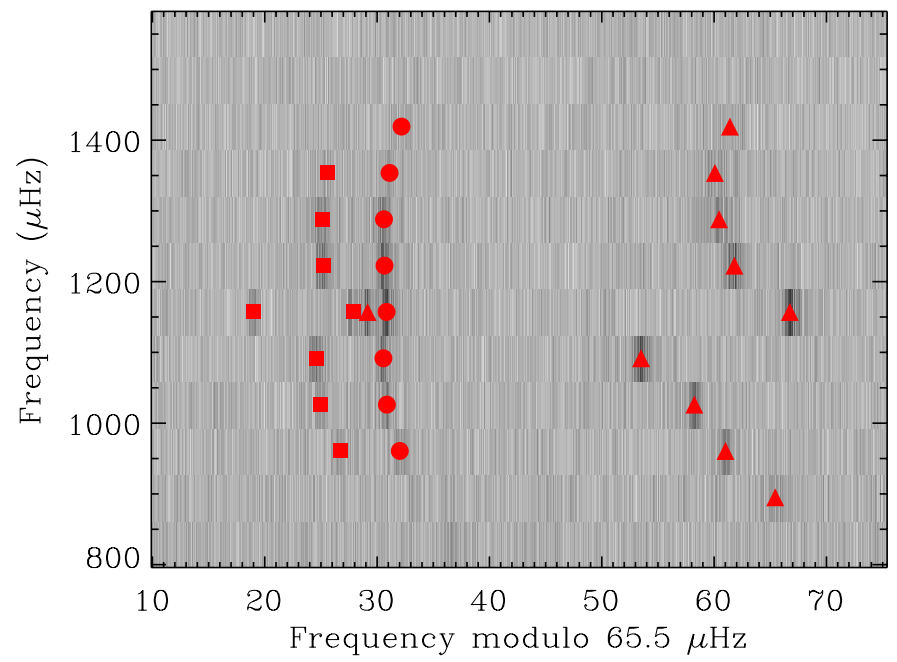

Fig. 4. Echelle diagram with the identified modes for KIC 11137075. The circles, triangles and squares show the frequencies of modes with $l=0,1$ and 2 .

of KIC 11137075 were not available, we chose the candidate models with $\chi_{v \text {,all }}^{2}<2000$ for this star, as listed in Table 5. We note that the Brunt-Väisälä frequency affects the distributions of oscillation modes undergoing avoided crossings. Models with similar global properties can have different mixed mode frequencies as a result of the diverse positions of avoided crossings. This causes in the large $\chi_{v, \text { all }}^{2}$ for some models listed in Table 5.

We note that the mass of these stars is not high enough to produce a convective core in the stellar interior, and core overshooting will not have any effect on their structure and evolution. Therefore, we do not discuss the effects of overshooting in the following part for these low-mass subgiants. 


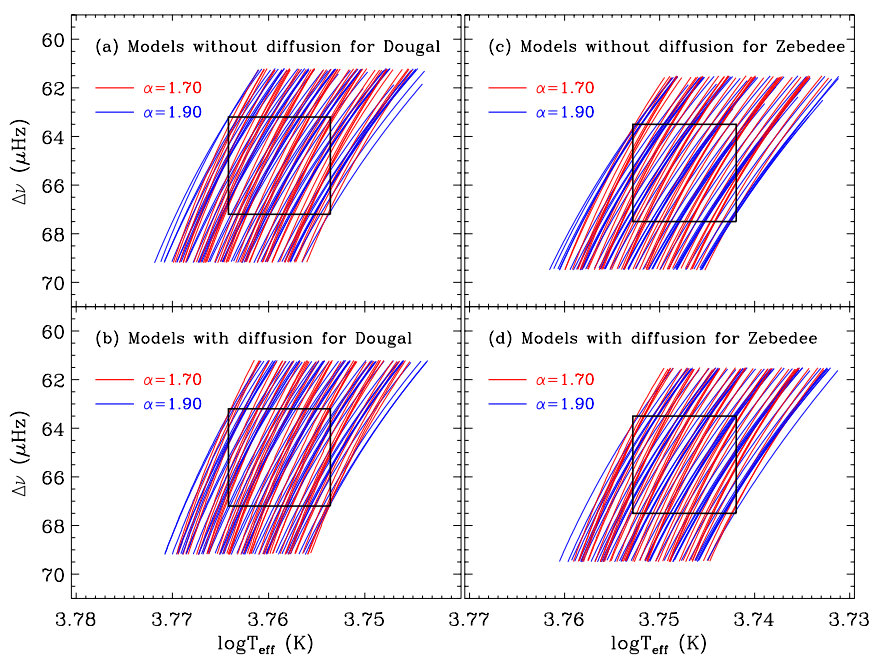

Fig. 5. Tracks for KIC 6442183 falling into the error box in the H-R diagram. The left and right panels denote models for KIC 6442183 and KIC 11137075, respectively. Red and blue colors in each panel denote models with mixing-length parameter $\alpha=1.70$ and $\alpha=1.90$, respectively.

\subsection{Optimal models}

The frequency separation between consecutive modes varies rapidly during an avoided crossing and so the mixed modes together with pure $p$-modes provide an important constraint to the stellar evolutionary state (Christensen-Dalsgaard et al. 1995; Pamyatnykh et al. 2004). We compared the frequencies and inertia ratios from observations and theoretical models listed in Table 5. The theoretical inertias for models were calculated using Eq. (4), and the observed inertia ratio through the following function (e.g. Benomar et al. 2014):

$\frac{I_{1}}{I_{0}}=V_{1} \frac{A_{0}}{A_{1}} \sqrt{\frac{\Gamma_{0}}{\Gamma_{1}}}$,

where $A$ and $\Gamma$ denote the amplitude and linewidth of modes, respectively, while the visibility $V_{1}$ is the square root of the ratio between theoretical heights for the dipole and radial modes. We adopted the observed amplitude, linewidth and the value of $V_{1}$ $\left(V_{1}^{2}=1.52\right)$ from Benomar et al. (2013) to deduce the observed inertia ratios.

In solar-like stars, $p$-mode oscillations are expected to follow the approximate relation (Tassoul 1980)

$v_{n, l} \approx\left(n+\frac{l}{2}+\epsilon\right) \Delta v-l(l+1) D_{0}$,

where $D_{0}$ is related to the interior structure of the star, and the offset $\epsilon$ is sensitive to the surface layers. The frequencies of the $p$-modes with the same degree show vertical ridges in the échelle diagram. The mean distance between dipole modes and radial modes with the same order would be $0.5 \Delta v-2 D_{0}$, which is deduced from the approximate relation formulated in Eq. (9). For red giants, Mosser et al. (2011b) introduced the expression for the pure $p$-mode eigenfrequency pattern:

$v_{n_{p}, l}=\left(n_{p}+\frac{l}{2}+\epsilon\right) \Delta v-l(l+1) D_{0}+\frac{\alpha}{2}\left(n_{p}-n_{\max }\right)^{2} \Delta v$

where $n_{p}$ is the $p$-mode radial order, $n_{\max }$ is the order of the mode with highest power and $\alpha$ is a constant representing the mean curvature of the $p$-mode oscillation pattern. Although the dipole

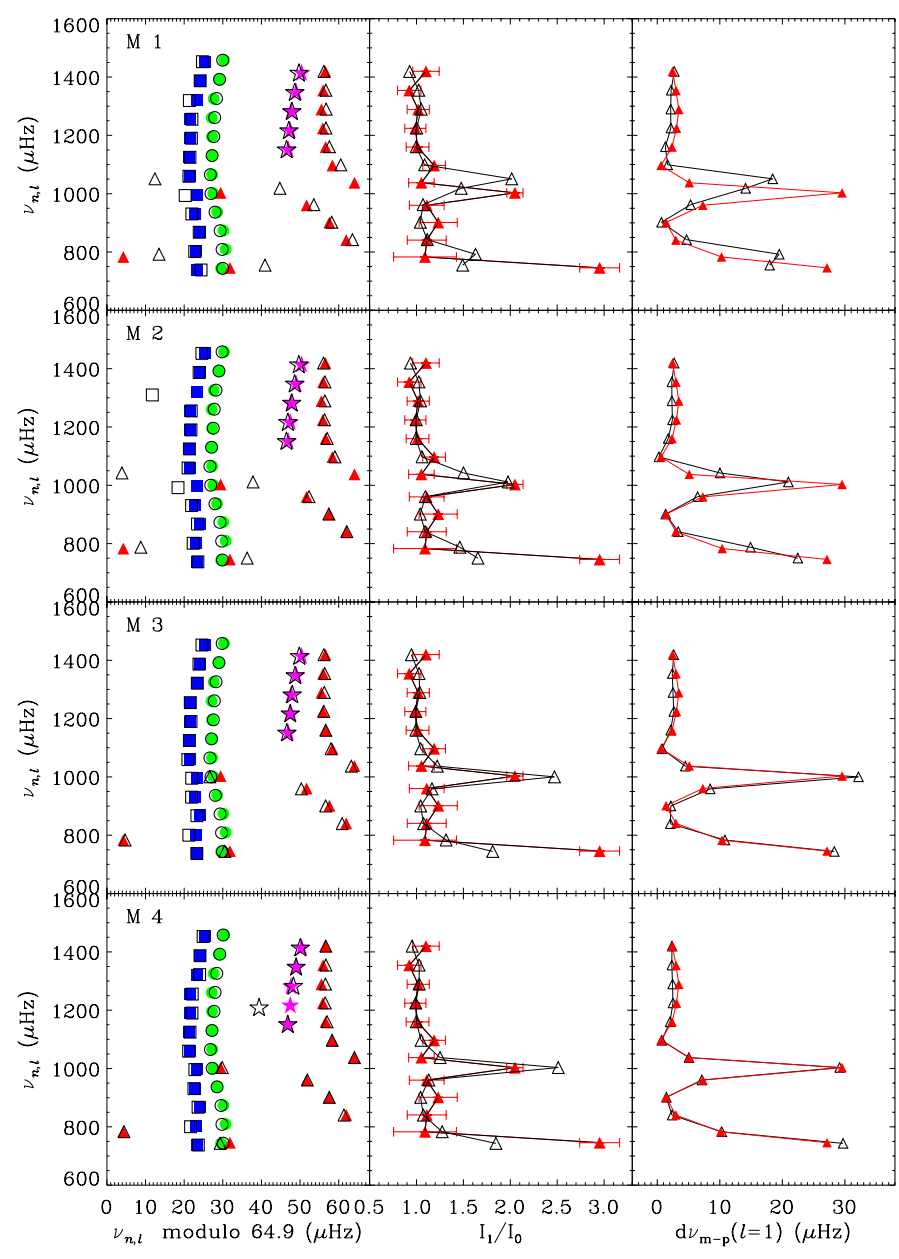

Fig. 6. Comparison of echelle diagram, inertia ratios, and $d v_{m-p}$ from observations and models without diffusion for KIC 6442183. The colorfilled symbols denote the observed frequencies, and the empty symbols are the corrected theoretical frequencies. The squares denote the $l=$ 2 modes, while the circles show the $l=0$, the triangle the $l=1$, and the five-pointed star the $l=3$ modes. We did not plot the frequency error bars since these are smaller than the symbol signs.

modes are $p-g$ mixed modes in subgiants, we were able to estimate the expected pure acoustic modes $v_{n_{p}, 1}=v_{n_{p}, 0}+0.5 \Delta v-2 D_{0}$ through Eqs. (9) or (10).

We defined a quantity $d v_{m-p}$ to measure the frequency difference between the mixed-mode frequency $v_{n, 1}$ and the pure acoustic modes $v_{n_{p}, 1}$. The quantity $d v_{m-p}$ for dipole modes was calculated using the following formula:

$d v_{m-p}=\left|v_{n, 1}-\left(v_{n_{p}, 0}+0.5 \Delta v-2 D_{0}\right)\right|$.

The $d v_{m-p}$ is the frequency difference between the mixed modes and the nearest $p$-mode. For $g$-dominated mixed modes, the higher the $Q_{n l}$, the stronger the coupling between $p$ - and $g$-modes. We used inertia ratios and $d v_{m-p}$ as criteria to constrain the stellar models of KIC 6442183 and KIC 11137075.

Through comparing theoretical and observed inertia ratios and $d v_{m-p}$ for KIC 6442183, we found that Models 3 and 4 without diffusion and Model 8 with diffusion match the observations better than the other models. We estimated the following parameters for KIC 6442183: $M=1.04_{-0.04}^{+0.01} M_{\odot}, R=1.66_{-0.02}^{+0.01} R_{\odot}$ and $t=8.65_{-0.06}^{+1.12}$ Gyr. By comparing Models 4 and 6, we found that the $T_{\text {eff }}$ and luminosity estimated from the model without diffusion are higher than those estimated from diffusion models, 


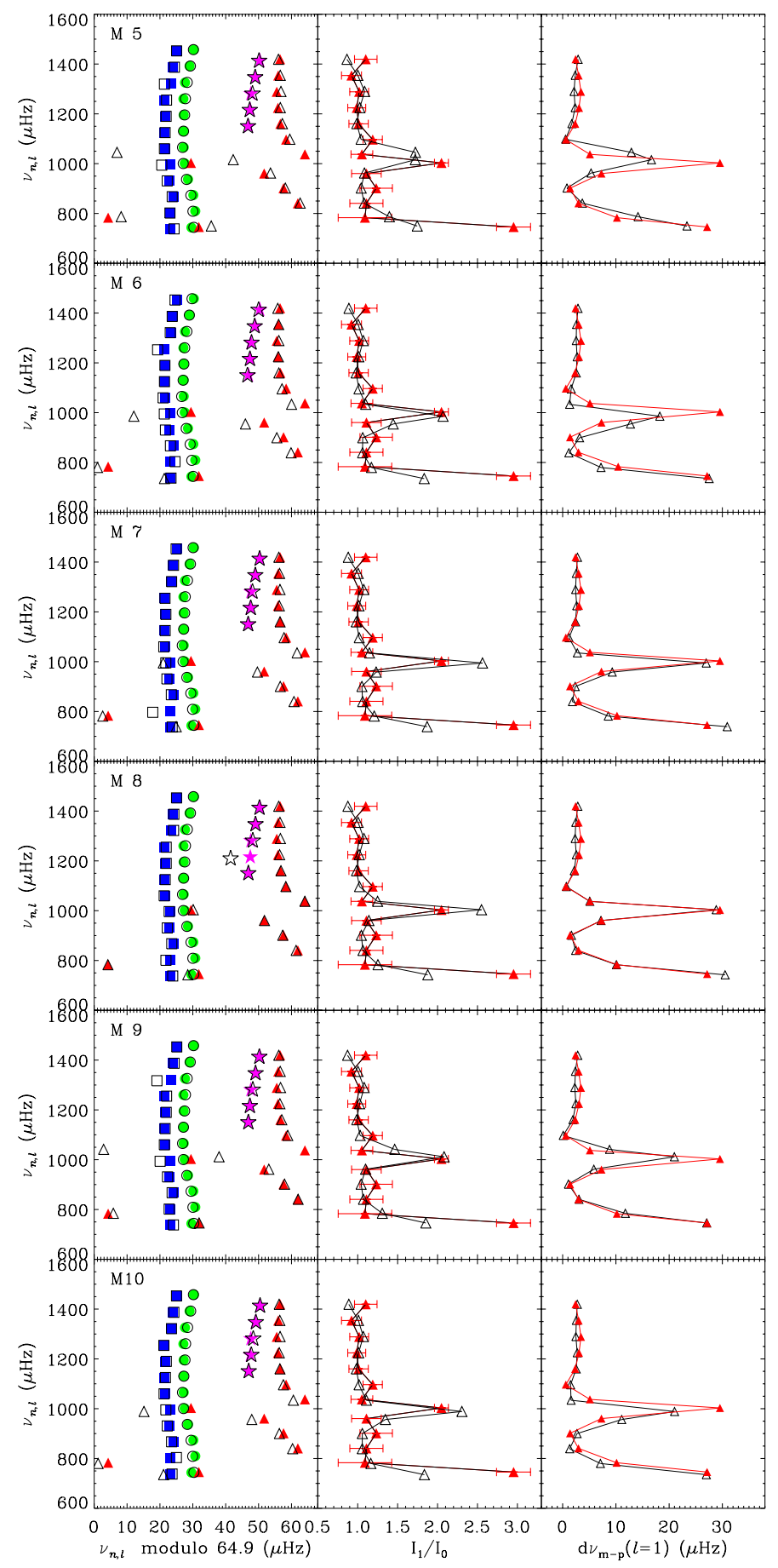

Fig. 6. continued.

which suggests that helium and heavy elements diffusions are not negligible. This is because the diffusion changes the opacity and equation of state for the models. The mass we find is approximately $11 \%$ higher than that deduced by Benomar et al. (2014) with the scaling relation.

In Fig. 7, we show the results for KIC 11137075. As shown in the figure, the maximum of theoretical dipole $d v_{m-p}$ is lower than the observations. For the $l=2$ models presented in the third column of Fig. 7, Models 3 and 5 without diffusion and Model 8 with diffusion match the observations much better than the other four models, which means that the three models reproduce the $l=2$ avoided crossing better than the other four models.

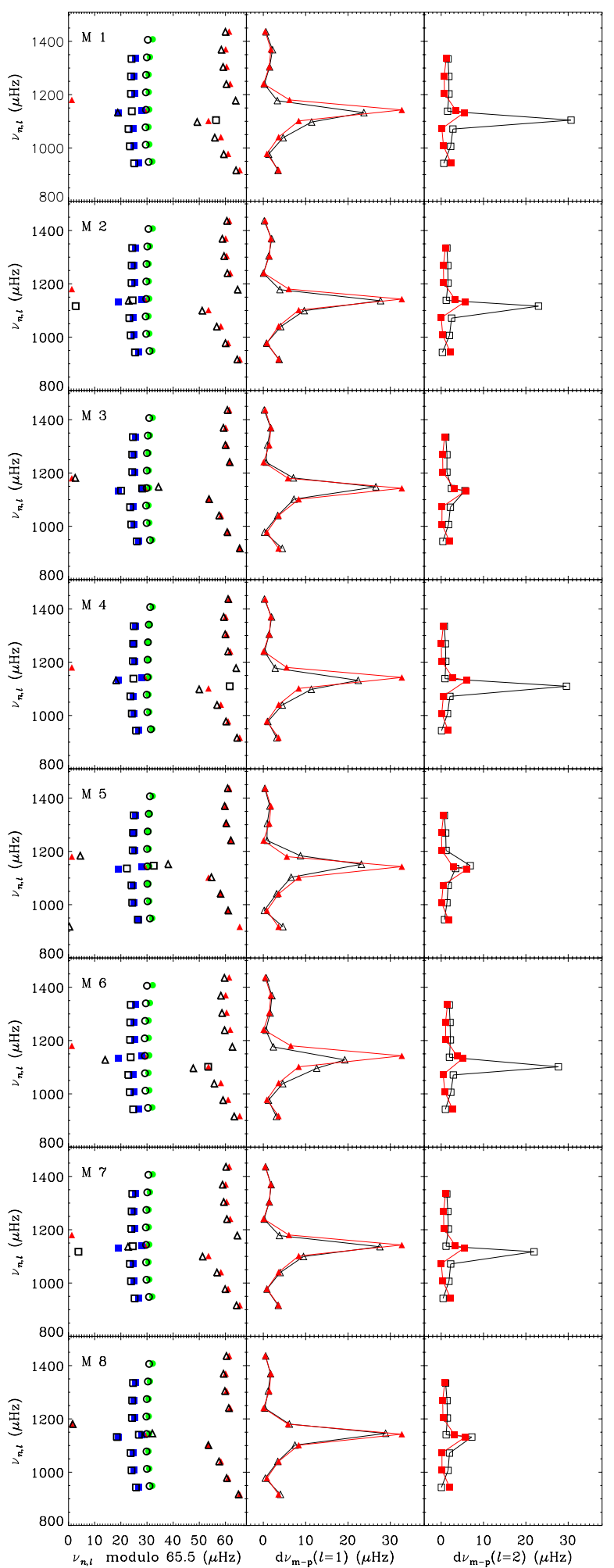

Fig. 7. Comparison of echelle diagram and $d v_{m-p}$ for observations and modeling for KIC 11137075 . The color-filled symbols denote the observed frequencies, and the empty symbols are the corrected theoretical frequencies. The squares denote the $l=2$ modes, while the circles the $l=0$ and the triangles the $l=1$ modes.

Thus, Models 3, 5, and 8 were selected as the best models for KIC 11137075 . Finally, we obtained the following parameters for KIC 11137075: $M=1.00_{-0.01}^{+0.01} M_{\odot}, R=1.63_{-0.01}^{+0.01} R_{\odot}$, and 
$t=10.36_{-0.20}^{+0.01}$ Gyr. The models with the mixing length $\alpha=1.90$ reproduce the observations better than the models with $\alpha=1.70$.

\section{Discussions and conclusions}

We carried out data processing and performed seismic analyses for subgiants KIC 6442183 and KIC 11137075 , which were observed by the Kepler mission. The main results and discussions are summarized as follows.

We applied the Lomb-Scargle periodogram to the corrected short-cadence time series observed by Kepler to obtain the power spectra, and estimated the large frequency separation and frequency of maximum power for the two stars: $64.9 \pm 0.2 \mu \mathrm{Hz}$ and $1225 \pm 17 \mu \mathrm{Hz}$ for KIC 6442183, and $65.5 \pm 0.2 \mu \mathrm{Hz}$ and $1171 \pm 8 \mu \mathrm{Hz}$ for KIC 11137075 . Individual mode frequencies were also extracted. After carrying out the asteroseismic analysis, we estimated the stellar parameters $M=1.04_{-0.04}^{+0.01} M_{\odot}$, $R=1.66_{-0.02}^{+0.01} R_{\odot}$ and $t=8.65_{-0.06}^{+1.12} \mathrm{Gyr}$ for KIC 6442183, and $M=1.00_{-0.01}^{+0.01} M_{\odot}, R=1.63_{-0.01}^{+0.01} R_{\odot}$ and $t=10.36_{-0.20}^{+0.01} \mathrm{Gyr}$ for KIC 11137075 . Both the subgiants are shown to be solar-mass stars.

For stars whose mass is similar to the Sun's, the helium and heavy-elements diffusion will affect the opacity and equation of state, which will change the thermal structure and affect the evolution of stars. Therefore, we have to consider the diffusion process in stellar models and seismic analysis.

Pure $p$-mode frequencies decrease and $g$-mode frequencies increase with age for subgiants (Christensen-Dalsgaard et al. 1995), which results in the change of the frequency at which the avoided crossing occurs (Bedding 2014). The frequencies of mixed modes that are sensitive to stellar interiors are useful to constrain stellar structures, especially for stellar interiors. The maximum of $d v_{m-p}$ is coincident with the high inertia ratio, allowing us to locate the mixed modes with the strongest $g$-mode property through these quantities. We first constrained stellar models with inertia ratios and $d v_{m-p}$, which provides an effective way to accurately determine the parameters of evolved stars.

In future work, we plan to select some evolved stars with mixed modes such as subgiants, red giants, and red clump stars, which constitute an evolution sequence. Then we aim to compare the differences of inertia ratios and $d v_{m-p}$ among these stars and use these quantities to constrain the stellar interiors with the quantities.

Acknowledgements. We are grateful to the entire Kepler team. This work was supported by grants 10933002, 11273007 and 11273012 from the National Natural Science Foundation of China, and the Fundamental Research Funds for the Central Universities.

\section{References}

Aerts, C., Christensen-Dalsgaard, J., \& Kurtz, D. W. 2010, Asteroseismology, 1 st edn. (Springer)

Aizenman, M., Smeyers, P., \& Weigert, A. 1977, A\&A, 58, 41

Appourchaux, T., Gizon, L., \& Rabello-Soares, M.-C. 1998a, A\&AS, 132, 107

Appourchaux, T., Rabello-Soares, M.-C., \& Gizon, L. 1998b, A\&AS, 132, 121 Appourchaux, T., Michel, E., Auvergne, M., et al. 2008, A\&A, 488, 705

Appourchaux, T., Benomar, O., Gruberbauer, M., et al. 2012, A\&A, 537, A134 Bahcall, J. N., Pinsonneault, M. H., \& Wasserburg, G. J. 1995, Rev. Mod. Phys., 67,781
Barban, C., Deheuvels, S., Baudin, F., et al. 2009, A\&A, 506, 51

Basu, S., Turck-Chièze, S., Berthomieu, G., et al. 2000, ApJ, 535, 1078

Bedding, T. R. 2014, Asteroseismology, 22nd Canary Islands Winter School of Astrophysics (Cambridge: Cambridge University Press), 60

Bedding, T. R., Mosser, B., Huber, D., et al. 2011, Nature, 471, 608

Benomar, O., Bedding, T. R., Stello, D., et al. 2012, ApJ, 745, L33

Benomar, O., Bedding, T. R., Mosser, B., et al. 2013, ApJ, 767, 158

Benomar, O., Belkacem, K., Bedding, T. R., et al. 2014, ApJ, 781, L29

Bi, S.-L., Basu, S., \& Li, L.-H. 2008, ApJ, 673, 1093

Böhm-Vitense, E. 1958, Z. Astrophys, 46, 108

Borucki, W. J., Koch, D. G., Lissauer, J., et al. 2007, in Transiting Extrapolar Planets Workshop, eds. C. Afonso, D. Weldrake, \& T. Henning, ASP Conf. Ser., 366, 309

Brandão, I. M., Doğan, G., Christensen-Dalsgaard, J., et al. 2011, A\&A, 527, A37

Brown, T. M., Gilliland, R. L., Noyes, R. W., \& Ramsey, L. W. 1991, ApJ, 368, 599

Bruntt, H., Basu, S., Smalley, B., et al. 2012, MNRAS, 423, 122

Chaplin, W. J., Houdek, G., Karoff, C., Elsworth, Y., \& New, R. 2009, A\&A, $500, \mathrm{~L} 21$

Chaplin, W. J., Appourchaux, T., Elsworth, Y., et al. 2010, ApJ, 713, L169

Christensen-Dalsgaard, J. 2004, Sol. Phys., 220, 137

Christensen-Dalsgaard, J., Bedding, T. R., \& Kjeldsen, H. 1995, ApJ, 443, L29

Datta, A., Mazumdar, A., Gupta, U., \& Hekker, S. 2015, MNRAS, 447, 1935

Demarque, P., Guenther, D. B., Li, L. H., Mazumdar, A., \& Straka, C. W. 2008, Ap\&SS, 316, 31

Dotter, A., Chaboyer, B., Jevremović, D., et al. 2008, ApJS, 178, 89

Dupret, M.-A., Belkacem, K., Samadi, R., et al. 2009, A\&A, 506, 57

Dziembowski, W. A., Gough, D. O., Houdek, G., \& Sienkiewicz, R. 2001, MNRAS, 328, 601

Eggenberger, P., Charbonnel, C., Talon, S., et al. 2004, A\&A, 417, 235

Ferguson, J. W., Alexander, D. R., Allard, F., et al. 2005, ApJ, 623, 585

García, R. A., Mathur, S., Salabert, D., et al. 2010, Science, 329, 1032

García, R. A., Hekker, S., Stello, D., et al. 2011, MNRAS, 414, L6

Gilliland, R. L., Brown, T. M., Christensen-Dalsgaard, J., et al. 2010a, PASP, 122,131

Gilliland, R. L., Jenkins, J. M., Borucki, W. J., et al. 2010b, ApJ, 713, L160

Guenther, D. B. 1994, ApJ, 422, 400

Huber, D., Stello, D., Bedding, T. R., et al. 2009, Comm. Asteroseismol., 160, 74

Iglesias, C. A., \& Rogers, F. J. 1996, ApJ, 464, 943

Kallinger, T., Weiss, W. W., Barban, C., et al. 2010, A\&A, 509, A77

Kjeldsen, H., \& Bedding, T. R. 1995, A\&A, 293, 87

Kjeldsen, H., Bedding, T. R., \& Christensen-Dalsgaard, J. 2008, ApJ, 683, L175

Kjeldsen, H., Christensen-Dalsgaard, J., Handberg, R., et al. 2010, Astron. Nachr., 331, 966

Lomb, N. R. 1976, Ap\&SS, 39, 447

Markwardt, C. B. 2009, Astronomical Data Analysis Software and Systems XVIII, 411, 251

Markwardt, C. 2012, Astrophysics Source Code Library, 1208.019

Mathur, S., Handberg, R., Campante, T. L., et al. 2011, ApJ, 733, 95

Metcalfe, T. S., Monteiro, M. J. P. F. G., Thompson, M. J., et al. 2010, ApJ, 723, 1583

Molenda-Żakowicz, J., Sousa, S. G., Frasca, A., et al. 2013, MNRAS, 434, 1422

Montalbán, J., Miglio, A., Noels, A., et al. 2013, ApJ, 766, 118

Mosser, B., \& Appourchaux, T. 2009, A\&A, 508, 877

Mosser, B., Barban, C., Montalbán, J., et al. 2011a, A\&A, 532, A86

Mosser, B., Belkacem, K., Goupil, M. J., et al. 2011b, A\&A, 525, L9

Mosser, B., Benomar, O., Belkacem, K., et al. 2014, A\&A, 572, L5

Osaki, J. 1975, PASJ, 27, 237

Régulo, C., \& Roca Cortés, T. 2007, A\&A, 469, 233

Pamyatnykh, A. A., Handler, G., \& Dziembowski, W. A. 2004, MNRAS, 350, 1022

Rogers, F. J., \& Nayfonov, A. 2002, ApJ, 576, 1064

Roxburgh, I. W. 2009, A\&A, 506, 435

Roxburgh, I. W., \& Vorontsov, S. V. 2006, MNRAS, 369, 1491

Scargle, J. D. 1982, ApJ, 263, 835

Tassoul, M. 1980, ApJS, 43, 469

Thompson, B., Frinchaboy, P., Kinemuchi, K., Sarajedini, A., \& Cohen, R. 2014, AJ, 148, 85

Thoul, A. A., Bahcall, J. N., \& Loeb, A. 1994, ApJ, 421, 828

Tian, Z. J., Bi, S. L., Yang, W. M., et al. 2014, MNRAS, 445, 2999 